Effective management of acute faecal incontinence in hospital: review of continence management systems Acute faecal incontinence in hospitals is a common problem that leads to misery and considerable morbidity. This review highlights the issues and provides practical solutions to minimise this distressing problem and advice on the use of faecal incontinence management systems. It is an essential read for those responsible for hospital-wide policies and procedures for patients with faecal incontinence, and anyone else looking after patients with this problem.

See page 94

Surveillance of Barrett's oesophagus: do we yet know whether it is worthwhile?

Surveillance of Barrett's oesophagus is a topic that generates diverse views and an intervention that uses valuable endoscopic resource. This evidence based review updates and reinforces the view that surveillance is difficult to justify. Perhaps things will change as new, less invasive, methods of treating Barrett's become mainstream?

See page 88

\section{Capsule endoscopy:}

10 years on and in the frontline

The UK was slow to adopt this British/ Israeli invention. A familiar story, which it is hoped this timely review will help rectify. Dr McAlindon and colleagues describe the circumstances in which capsule endoscopy enhances patient management and look to the future potential of pangut capsule endoscopy.

See page 82

The emerging role of screen based simulators in the training and assessment of colonoscopists

I am frequently asked about the role of simulators in training and this review provides the answer.

See page 76

Revalidation for gastroenterologists, with or without sedation!

Revalidation has arrived, and this article describes what it means and what consultants have to do to comply with current recommendations. It is, quite simply, essential reading for all consultants-except of course those who are due to retire and who are, undoubtedly, smiling as they reach for their pension.

See page 70

High resolution manometry and multichannel intraluminal impedance oesophageal manometry in clinical practice

Testing oesophageal function is now part of mainstream gastroenterology and no longer the preserve of research laboratories or those with a 'special interest'. This review describes the latest technology and how it might be applied in the clinical setting.

See page 112

\section{Malnutrition and nutrition support in patients with liver disease}

Treating malnourished patients with chronic liver disease has always seemed a hopeless task. This review illustrates that this is not the case, providing a logical approach to assessment and treatment. Malnutrition in liver disease is common-it is no longer acceptable to ignore it.

See page 105

\section{Abnormal liver function tests in the parenteral nutrition fed patient}

Parenteral nutrition has revolutionised the management of intestinal failure but it is not without problems. Abnormal liver function tests can be related to the treatment or other reversible causes. This review provides a practical framework for managing abnormal LFTs in association with parenteral nutrition.

See page 98 